\title{
Updating Geologic Models Using Ensemble Kalman Filter for Water Coning Control
}

\author{
Cesar A. Mantilla, Sanjay Srinivasan, Quoc P. Nguyen \\ The University of Texas at Austin, Austin, USA \\ E-mail:sanjay.srinivasan@engr.utexas.edu \\ Received February 5, 2011; revised March 15, 2011; accepted April 19, 2011
}

\begin{abstract}
This study investigated the feasibility of updating prior uncertain geologic models using Ensemble Kalman filter for controlling water coning problems in horizontal wells. Current downhole data acquisition technology allows continuous updating of the reservoir models and real-time control of well operations. Ensemble Kalman Filter is a model updating algorithm that permits rapid assimilation of production response for reservoir model updating and uncertainty assessment. The effect of the type and amount of production data on the updated geologic models was investigated first through a synthetic reservoir model, and then implemented on a laboratory experiment that simulated the production of a horizontal well affected by water coning. The worth of periodic model updating for optimized production and oil recovery is demonstrated.
\end{abstract}

Keywords: Ensemble Kalman Filter, Reservoir Model Updating, Oil Production Optimization

\section{Introduction}

The productive life of a well completed over an active aquifer is strongly affected by water coning. The evolution of the water cone is driven by non-uniform drawdown pressure near the wellbore. The irreversibility of water coning mandates its early detection, modeling and control before and/or during water breakthrough. Recent development in technologies for in-situ monitoring using pressure and temperature distributed sensors allow acquisition of real-time production data that carry valuable information about reservoir properties and the water conning process in the vicinity of the well. The acquired data can be used to maximize oil-water ratio through model based dynamic optimal control of pressure drawdown along a production well and/or inflow allocation among the wells draining the same reservoir.

Water coning is a local phenomenon governed by the gradient of the flow potential in the vicinity of the well. Since the flux variations in the far-field have a relatively insignificant influence on the characteristics of coning, a full reservoir model is not required to control this local phenomenon. Muskat [1] presented an analytical solution for the critical flow rate of a well in a homogeneous and isotropic reservoir below which a stable water cone is formed. The velocity of cone propagation is partially determined by reservoir permeability as shown in (1).

$$
\frac{\partial h}{\partial t} \propto \Delta P_{t o t}=\frac{\mu}{2 \pi k D} \ln \left[\frac{r_{o}}{b(1-h)}\right]-\Delta \rho g b h-2 P_{c}
$$

$h$ is Cone height from original water-oil contact, $\Delta P$ is the pressure difference from the tip of the cone to the wellbore, $\mu$ is the oil viscosity, $k$ the average permeability, $b$ is the distance from the well to original water-oil contact, $\Delta \rho$ is the oil-water density difference, $g$ is the gravity force, and $P_{c}$ is the capillary pressure.

Several analytical and numerical correlations that predict water coning performance accounting for more complex geometries and heterogeneity have been developed. A comprehensive literature survey on water coning was published by Alikhan and Ali [2]. Despite the extensive work done on water coning modeling, most of the existing models for this process are deterministic in the sense that the reservoir properties (such as permeability $k$ in (1)) are assumed to be known. However, in most cases, the knowledge of reservoir properties is limited, and uncertainty in model parameters causes deviations in the prediction of these physical quantities. Updating of reservoir properties based on acquired dynamic data is crucial. Conventional history matching techniques may not be appropriate for this purpose since they are computationally expensive and time-consuming for real-time integration of data. Ensemble Kalman filter has demonstrated to be a rapid tool for subsurface process identifi- 
cation and control. Ensemble Kalman filter (EnKF) has been applied for water flooding optimization $([3,4])$, uncertainty assessment in reservoir description [5], history matching [6], seismic incorporation [7], and other areas.

In this paper, we present an iterative form of EnKF used in conjunction with dynamic process optimization. The effect of data type and its diversity on the adequacy of EnKF is first investigated through a synthetic case of water coning, and then demonstrated on a laboratory flow model. The results from this study provide a guideline for development of an effective monitoring system for real-time system characterization. The integration of such a monitoring system into an optimal process control system is also demonstrated through the lab-scale water conning problem.

A schematic flow chart summarizing the system model updating procedure is presented in Figure 1. This procedure is connected to an optimization module as part of a closed-loop feedback control system. The system, which is composed of the reservoir, wells and surface facilities is modeled using a reservoir simulator. Conventionally, the geologic model is constructed using petrophysical data such as permeability and porosity obtained from well logs and/or well tests. However, the inherent uncertainty in the distribution of these properties throughout the reservoir results in uncertain predictions of reservoir performance. EnKF enables a rapid geologic model updating for short-term or local problems such as water coning, and thus allow real-time optimal control of operating conditions.
A brief description of ensemble Kalman Filter is presented here; a detailed derivation of EnKF is presented by $\mathrm{Gu}$ and Oliver [4]. Denote $N R$ as the numbers of equally probable realizations of the distribution of an uncertain reservoir property (i.e. permeability $k$ in this study) and $N B$ as the number of gridblocks in the reservoir model. The ensemble of $N R$ realizations is generated from the prior information of the reservoir using sequential Gaussian simulation. Each realization is input into the reservoir simulator and run for one time step $\left(t_{i}\right)$, producing a set of NRes production data, denoted by the vector $\boldsymbol{d}$ (including oil, water, gas rates, etc.). The covariance between the NRes responses and the $N B$ grid block permeability can be calculated using the $N R$ realizations. This covariance matrix forms the basis for the Kalman Gain matrix $K \boldsymbol{G}$. The model represented by the vector $\boldsymbol{K}$ is updated using the following linear model:

$$
\boldsymbol{K}_{i}^{\text {new }}=\boldsymbol{K}_{i}^{\text {old }}+\boldsymbol{K} \boldsymbol{G} \times\left(\boldsymbol{d}_{\text {ref }}-\boldsymbol{d}_{i}\right)
$$

where $\boldsymbol{d}_{r e f}$ is the vector containing the set of production data of the real or reference reservoir, $\boldsymbol{K}^{\text {new }}$ is the vector with the updated variable corresponding to the $i^{\text {th }}$ realization, $\boldsymbol{K}^{\text {old }}{ }_{i}$ is the vector with the prior variable of the $i^{\text {th }}$ realization, $\boldsymbol{d}_{i}$ is the vector with the set of production data of the $i^{\text {th }}$ realization. The covariance matrix between the permeability values at each location and the production responses from the ensemble responses is formed. Then, each element of the covariance matrix is standardized by the variance of the production responses of the ensemble to compute the Kalman Gain matrix.

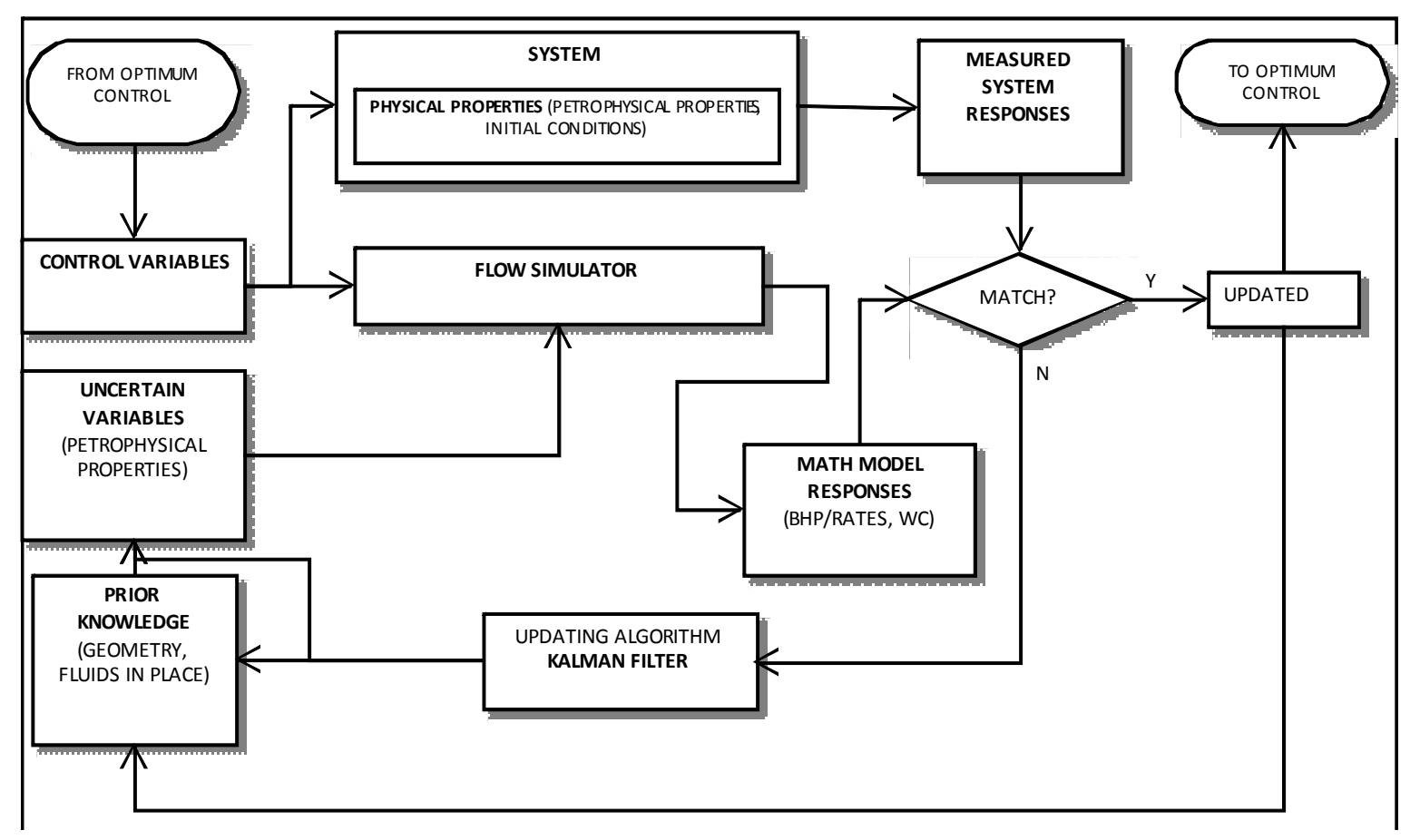

Figure 1. Model Updating flow chart. 
It is expected that the ensemble of updated models matches better with the true data. However, in practice when the updated models are again processed through the flow simulator, the corresponding responses continue to exhibit deviation from the measurements. This mismatch reflects the inadequacy of the covariance-driven updating scheme for capturing the non-linearity of the transfer function (simulator) model. In order to achieve a better match, the updating procedure is continued iteratively until a tolerable deviation is obtained. The whole process is then repeated for the next time step $\left(t_{k+1}\right)$. Once the deviation of updated model responses from the measured data is acceptable, it is transferred to the optimization module, and the well settings are re-evaluated to maximize the production for the remaining time. The wells are operated with the new operating conditions and the monitoring/data acquisition step is continued.

The type and amount of data included in the responses (d) plays an important role in the update of the uncertain variable $(\boldsymbol{K})$. More data assimilated not necessarily improve the estimation. If the responses are highly correlated to each other, as in the case of two downhole pressure sensors located too close to each other, they can bring redundant information with the natural noise associated to a measurement without improving the estimation. On the other hand, if few data are used, it becomes difficult to update the model properly. This type of sensitivity analysis would be useful to choose the best location of the sensors that improves the updating process without being redundant.

In this case, the types of data used are oil and water rates. Intuitively, each type of data carries information from different parts of the reservoir, for example water rates are correlated to properties of an associated aquifer. In other cases, data can be uncorrelated to the spatial variations in reservoir properties and hence model updated including such data will not necessarily improve the predictability of the model. A sensitivity analysis on the type of data is recommended to select the responses according to the model updating objectives.

\section{Synthetic Flow Model with a Horizontal Well}

\subsection{System Description}

A three dimensional rectangular synthetic reservoir was created to run sensitivity cases on the type and amount of data to be used for updating. Since water coning is a local phenomenon unaffected by the pressure conditions in the remaining reservoir, the near wellbore area is assumed surrounded by a constant pressure boundary condition. This is simulated by placing a set of oil injectors replenishing the oil produced by the producers. The grid is composed of $25 \times 20 \times 20$ blocks in $x, y$ and $z$ directions respectively. Local grid refinement is performed near the well. A constant porosity value equal to 0.1 is set. A horizontal well is completed in the middle of the layer and spans a length of six grid blocks in the $\mathrm{x}$ direction for a total length of $240 \mathrm{ft}$. The dimensionless well length $\left(\mathrm{L}_{\text {well }} / \mathrm{Lr}_{\text {eservoir }}\right)$ is 0.1 .

The well has 3 segments whose center points are regularly spaced $80 \mathrm{ft}$ apart. The well production is constrained by the bottomhole pressure in the toe $\left(P_{1}\right)$, and the bottomhole pressure at the other two segments $\left(P_{2}\right.$ and $P_{3}$ ) is calculated from frictional pressure gradient.

An active aquifer is beneath the oil reservoir, and the oil and water rate data for each segment are used for updating the prior geological model. The reservoir property to be updated is the permeability value in all grid-blocks which is spatially distributed using a semi-variogram with a range of 15 grid-blocks in $x$. The average permeability of the reference reservoir is $100 \mathrm{mD}$.

An initial suite of 40 realizations was generated using sequential Gaussian simulation sampling from a lognormal distribution with mean $180 \mathrm{mD}$ and standard deviation $50 \mathrm{mD}$. The permeability field was updated in the normal (log-transformed) space, so that it is guaranteed to be positive after back-transformation to the original space. As shown by Evensen [8], the distribution of the variable of interest in Ensemble Kalman Filter should be Normal for the updating equation to work properly.

\subsection{Results and Discussion Synthetic Flow Model}

Water broke in the well after 726 days of net oil production, initially through segment $P_{2}$. Water arrives first to the central perforation because the apex of the cone is centered within the well path, as observed on the saturation map shown in Figure 2. Once water breaks into $P_{2}$, the oil rate decreases as consequence of the reduction in the relative permeability to oil in that grid-block (Figure 3); the other two segments maintain the oil rate for a short time until water makes its way to those grid blocks. The total duration of the simulation was 1500 days. Although the reservoir simulator reports data every week, for simplicity only 5 time steps of 300 days each were used to update the model. Before water breakthrough the model was updated by only using oil rates. Subsequently water rates were also included as part of the sensitivity analysis.

Effect of data type. Oil and water rates are the two types of data available to update the model. The objective is to select the best data to update. Two cases were 
Side view of oil saturation at breakthrough

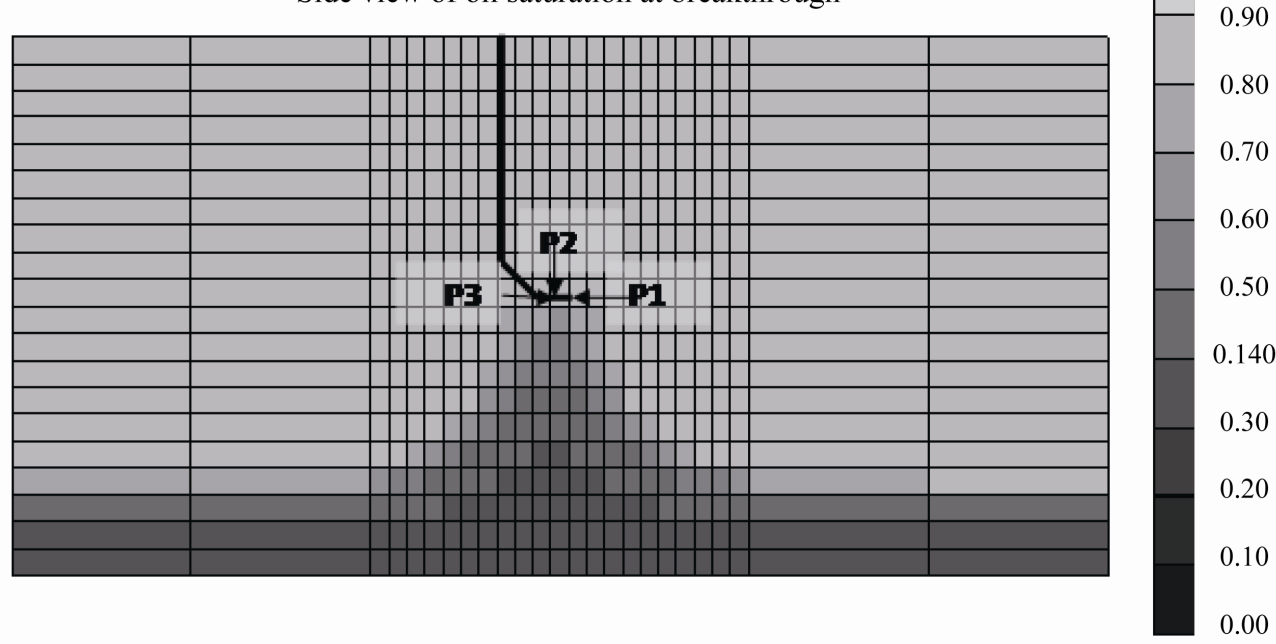

Figure 2. Profile of the water cone resulting in breakthrough at 726 days.s
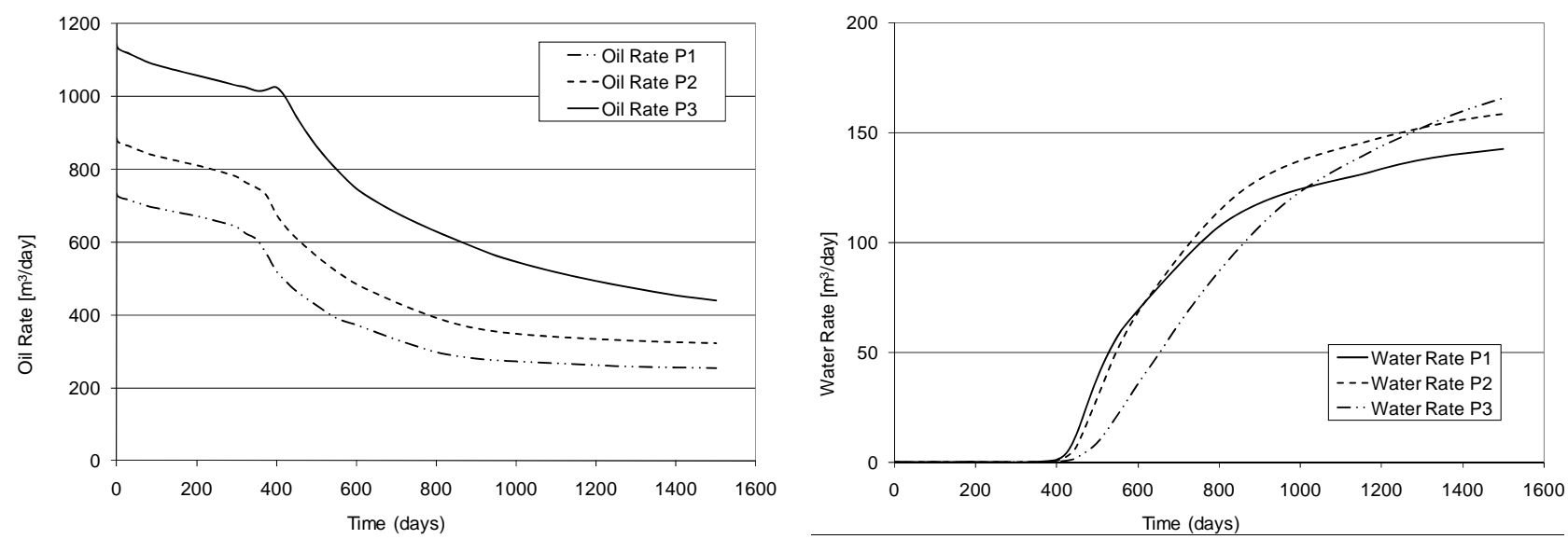

Figure 3. Oil production rates corresponding to the reference reservoir. Water breaks through initially in the well segment P2 and later in P1 and P3. Note that the reduction in the oil rate in P1 and P3 is less severe.

analyzed: in Case 1, water and oil rates from the 3 perforations were included in the objective function; and Case 2 only the oil rate from the 3 perforations were included, the water rate was excluded from the objective function. Oil and water rates from each well segment enter in the updating equation independently, and the model is updated until each segment rate (water and oil) satisfies the specified tolerance. In order to have a fair comparison, the total oil and water rates from the well (adding the three production points) are compared regardless whether they are used to update the model or not. The ultimate goal is to predict the total production of the well, not partial rates.

Predictability. The accuracy of the updated models in terms of the predictability of oil and water rates from Cases 1 and 2 are compared in Figure 4. Figure 4(a) shows the predicted oil rates for time step 3 (900 days), at this time both cases had the same predicted oil and water rates since in time steps 1 and 2 previous time steps they both used only oil rates to update because water had not breakthrough yet. After updating at 900 days, Case 2 resulted with oil rates closer to the reference because its objective was only to reduce the mismatch the oil rates as opposed to Case 1 , where the objective was to simultaneously reduce the mismatch in both oil and water rates. In Case 1, the minimization of mismatch of total (oil + water) rates causes some compromise to be made in honoring the oil rates alone.

Although Case 2 matched closer the oil rate, the mismatch in predicted water rate was higher than in Case 1 as shown in Figure 4(b). This is because Case 2 did not included water rates in the updating equation. In summary, Case 2 predicts better oil rates while Case 1 predicts water rates. However, Figure 5(a) shows that the 


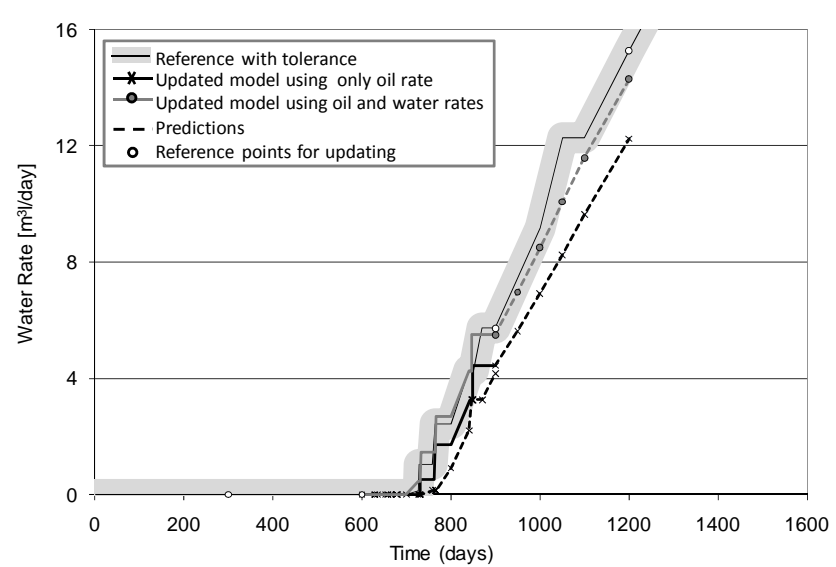

(a)

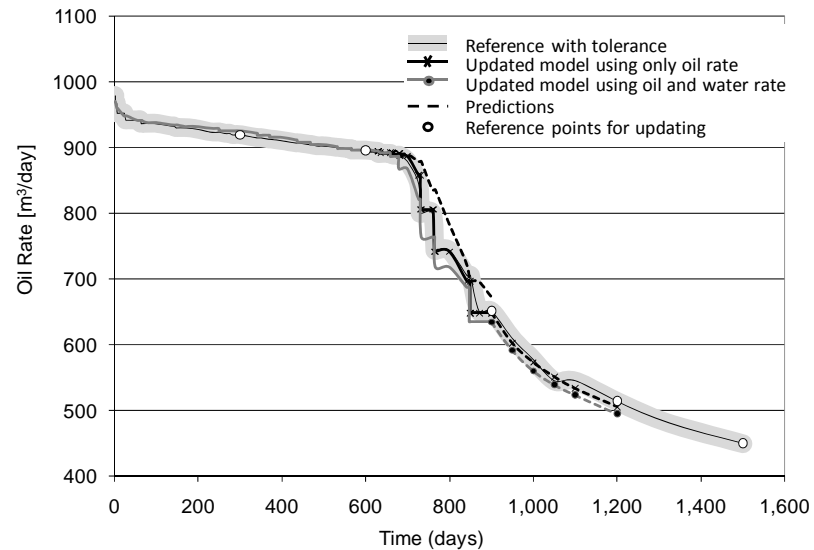

(b)

Figure 4. Prediction of (a) oil and (b) water rates with a model updated using both oil and water rates as compared to that using only oil rate.

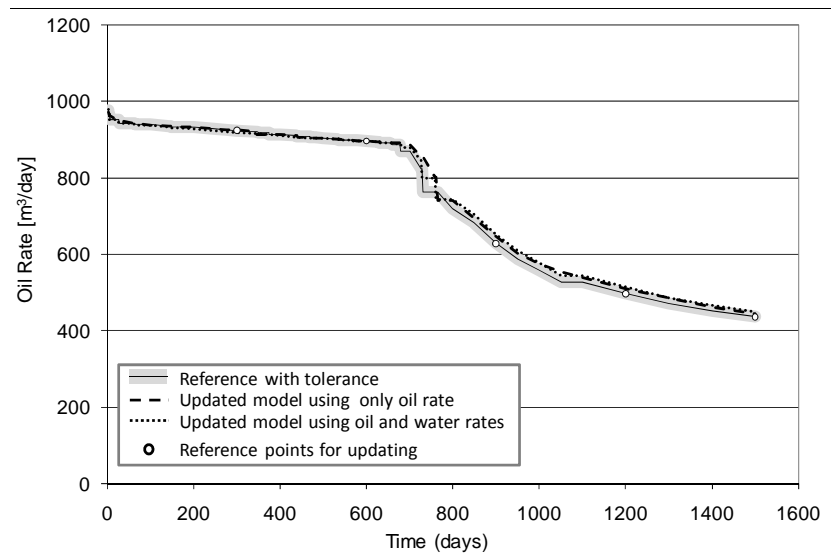

(a)

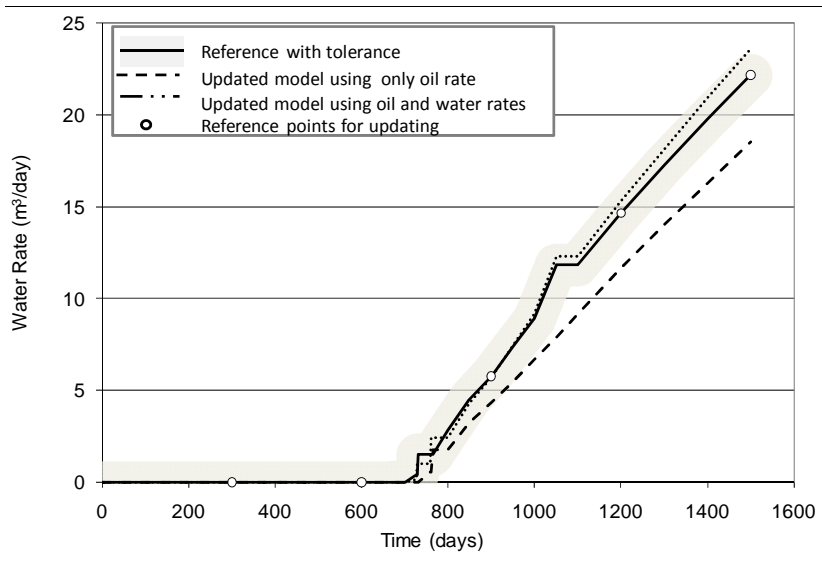

(b)

Figure 5. The match in (a) oil and (b) water rates over the entire simulation duration using a model updated using only the oil rate data as compared to that using total fluid rate data.

overall performance of both cases in terms of predicting the oil rate was not significantly different over the entire simulation duration. On the other hand, Figure 5(b) also indicates that the mismatch in terms of water rate becomes progressively worst for Case 2 . This has drastic consequences in terms of deriving and implementing an optimum control scheme.

Estimation of Permeability. The estimated permeability from Cases 1 and 2 is then compared to identify reservoir regions where the water rate improves the model updating. Figure 6 compares the deviation of updated model corresponding to Case 2 against the reference and indicates that the highest error in permeability prediction occurs in the aquifer and the water invaded zone because these directly affect the flow of water. On the other hand, Case 1 yields a better estimation of permeability in the water zone as shown in Figure 6(b).

Geological consistency in the updated models. The experimental variogram is a representation of the spatial variability and geological consistency exhibited by the updated model. Figure 7 compares the updated experimental variogram from Cases 1,2 and the reference; the range of the variogram in $x$ for the reference permeability distribution was 15 gridblocks. For each case, the permeability values of all realizations were averaged, and the variogram of the averaged ensemble was compared with the reference variogram. In Case 1, where water rates improved the estimation of permeability in the water zone, and the variogram is similar to the reference variogram. In comparison, the updated model for Case 2 exhibits a variogram that did not reach a sill. This lack of variogram reproduction and the associated difference in spatial characteristics of the models is what is responsible for the poor predictive capability of the models, especially in the Case 2 where the permeability in the water zone was poorly estimated. 


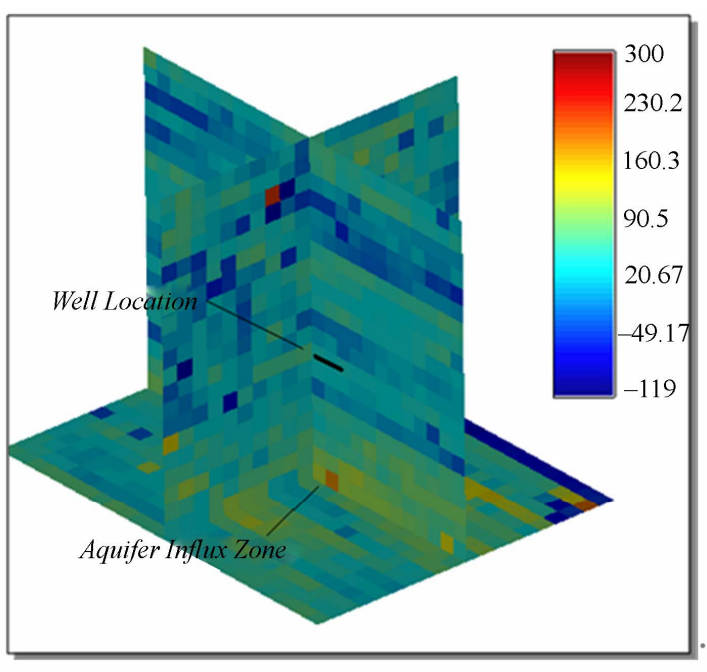

(a)

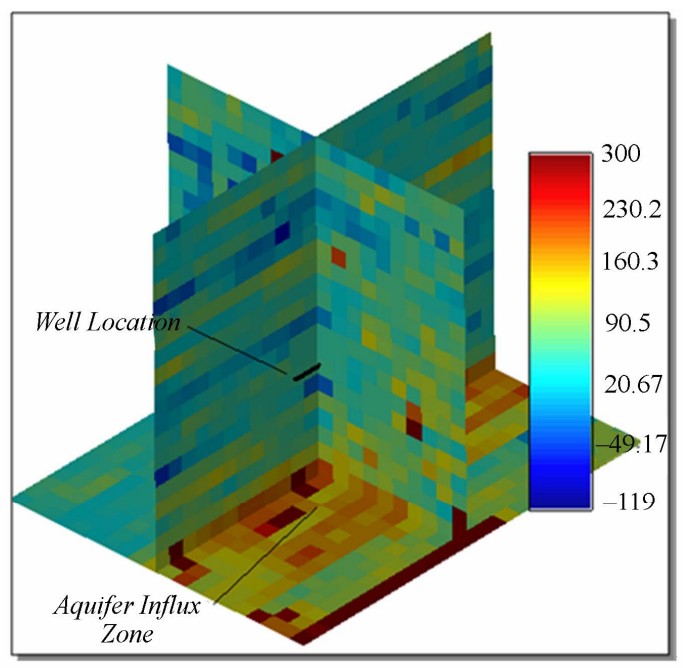

(b)

Figure 6. (a) Error map computed as model minus the reference for Case 1 . (b) Error map for Case 2. Results show that the permeability water zone was better estimated in Case 1.

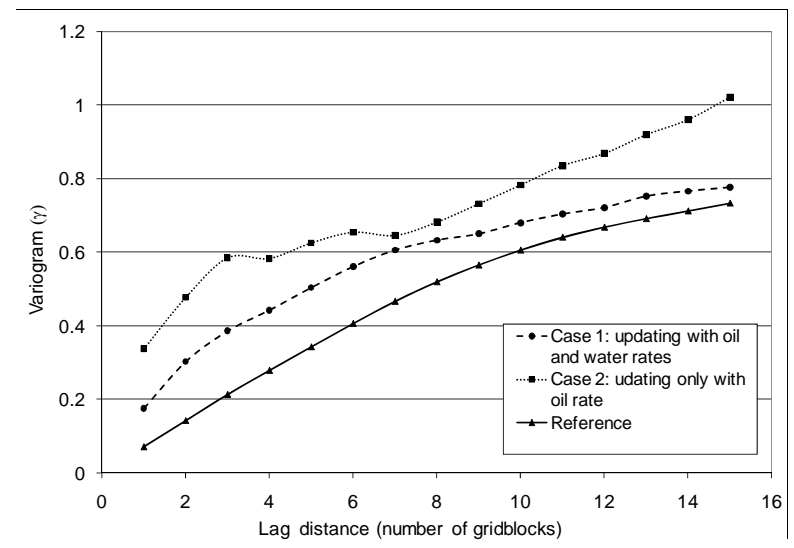

Figure 7. Variogram reproduction of the updated model indicates that Case 1 had similar characteristics as the reference, while the variogram for Case 2 is non-stationary.

\section{Laboratory 2D-Flow Model}

\subsection{Description of the Experiments}

A sand pack with glass beads and two layers of different grain sizes, and consequently two permeability layers was created. The top layer was packed with 0.3 to 0.43 $\mathrm{mm}$ beads corresponding to the low permeability layer and the bottom layer with 1.4 - $1.7 \mathrm{~mm}$ beads, corresponding to the high permeability layer. The total porosity was measured while the initial filling and assumed constant $(25.9 \%)$ after packing and shaking the apparatus. The main elements of the laboratory model are indicated in Figure 8. Oil was replenished from 6 injection points at the top of the reservoir at constant pressure maintained with air in a tank with decane (oil phase). These injection

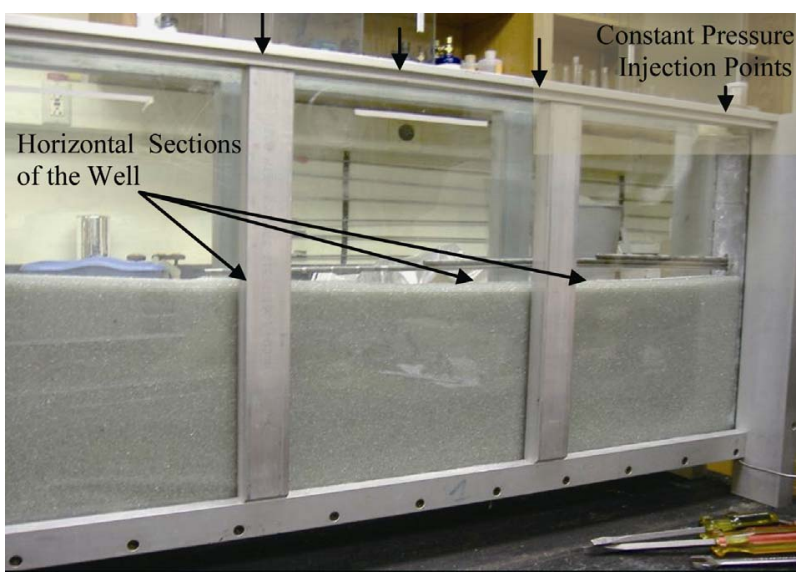

Figure 8. The laboratory 2D-flow model prior to packing the second layer of glass beads. A horizontal well spans through the center of the porous medium with two segments independently operated.

points provided a constant pressure boundary. Red dyed water was injected laterally to simulate an active aquifer at approximately the same constant pressure as the oil injection pressure. Oil flows through the low permeability layer whereas water flows through the high permeability layer, this was designed to promote water coning in the laboratory. A horizontal well, located inside the high permeable layer close to the interface with the low permeability layer, spans through the center of the reservoir with two independently segments controlled using electronically actuated inflow control valves

The main characteristics of a two-layer 2D reservoir with an active aquifer and a horizontal well at the center were simulated. Before conducting the experiments, the average permeability of each layer was estimated with a 
steady-state experiment. The estimated average permeability was 1100 and $6700 \mathrm{mD}$ for the top and the bottom layer respectively. The permeability of each layer was also calculated using Carman-Kozeny's equation resulting in $700 \mathrm{mD}$ and $10,700 \mathrm{mD}$. Though this estimation is not accurate because values for porosity and tortuosity were assumed, they are in the same range of the experimental measurement. With this prior information the initial ensemble of realizations was generated using sequential Gaussian simulation in each layer separately.

The glass pack was modeled in CMG's IMEX using the same dimensions as the laboratory prototype. The boundary conditions were (1) constant pressure oil injection at the top, (2) constant pressure water injection from the bottom left side and (3) the producers were pressure constrained according to the data recorded by the pressure transducers (Figure 9). Nine measurements of oil and water rates were collected over a 35-minute period for each well segment (Table 1). Pressure data recorded by the transducers from each segment at the times listed in Table 1 were used as the pressure constraints for the horizontal well in the simulation. Oil and water rates were measured by recording the weight and volume of the total liquids produced from each well segment.

In the laboratory prototype, oil production generated a pressure gradient towards the well, forming a uniform water cone with the tip at the center of the porous medium, which eventually broke through close to the center of the well. Figure 10(a) shows that water production began after seven minutes of clean oil production. The water cone invaded the production port $P_{2}$ more severely than $P_{1}$. After 17 minutes, water and oil rates became steady until the end of the experiment. Pressure data recorded by the transducers from each segment at the times listed in Table $\mathbf{1}$ were used as the pressure constraints
Table 1. Measured rate and pressure data during the water coning experiment

\begin{tabular}{ccccccc}
\hline Time & Oil P1 & Oil P2 & Wat.P1 & Wat.P2 & Press.P1 & Pres.P2 \\
\hline $\mathbf{m i n}$ & $\mathbf{c m}^{3} / \mathbf{m i n}$ & $\mathbf{c m}^{3}$ /min & $\mathbf{c m}^{3} / \mathbf{m i n}$ & $\mathbf{c m}^{3} / \mathbf{m i n}$ & KPa & KPa \\
7 & 339 & 188 & 1 & 0 & 128.06 & 122.84 \\
10.5 & 213 & 302 & 15 & 10 & 127.99 & 122.54 \\
14 & 231 & 225 & 22 & 38 & 127.43 & 122.16 \\
17.5 & 245 & 210 & 23 & 43 & 127.42 & 121.86 \\
21 & 240 & 210 & 23 & 46 & 126.88 & 121.70 \\
24.5 & 250 & 205 & 26 & 45 & 126.77 & 121.81 \\
28 & 250 & 200 & 26 & 46 & 126.49 & 121.58 \\
31.5 & 255 & 205 & 27 & 47 & 126.32 & 121.42 \\
35 & 260 & 205 & 28 & 44 & 126.32 & 121.35 \\
\hline
\end{tabular}

for the horizontal well in the simulation. Oil and water rates were measured by recording the weight and volume of the total liquids produced from each well segment.

\subsection{Updating the Sand-Pack Flow Model}

Fifty realizations were generated using sequential Gaussian simulation conditioned to the average permeability measured for each layer. Similar to the synthetic example, two cases were run. In Case 1 both water and oil rates from $P_{1}$ and $P_{2}$ were used to update the model, and in Case 2 only the oil rates were used. A model was accepted as updated if the mismatch in the prediction of the water and oil rates was less than $3 \mathrm{~cm}^{3} / \mathrm{min}$. After the ensemble was updated, the simulator predicted the oil and water rates of each well segment for the next time step. At each time step, the error in the predicted oil rate over the set of $N R$ realizations was calculated as:

$$
E_{o}=\left(\hat{q}_{o}^{P 1}+\hat{q}_{o}^{P 2}\right)-\frac{1}{N R} \sum_{i=1}^{N R} \hat{q}_{o, i}^{P 1}+\hat{q}_{o, i}^{P 2}
$$

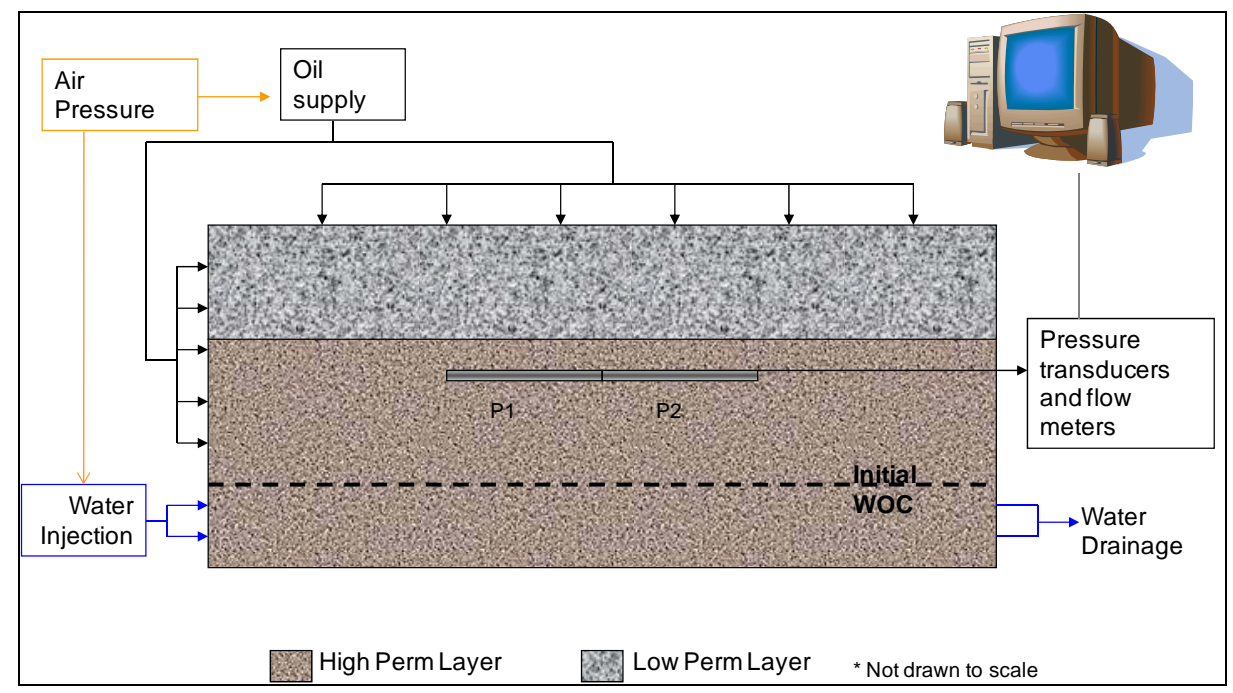

Figure 9. Laboratory porous media simulated in CMG. Location of injection and production wells is indicated. 


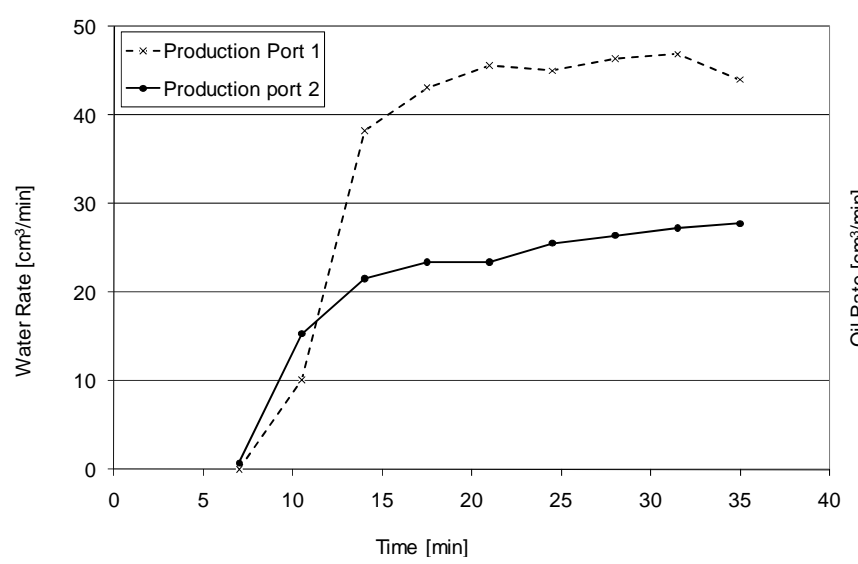

(a)

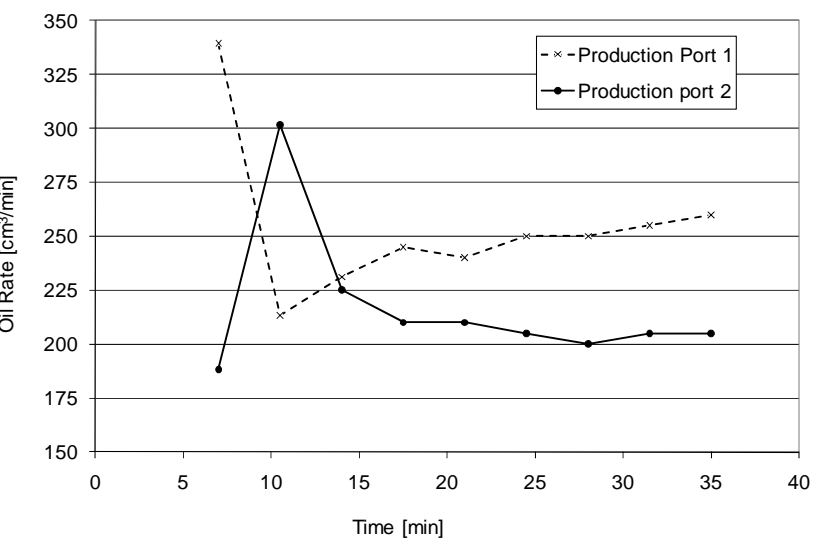

(b)

Figure 10. Measured (a) swater and (b) oil rates for each well segment P1 and P2.

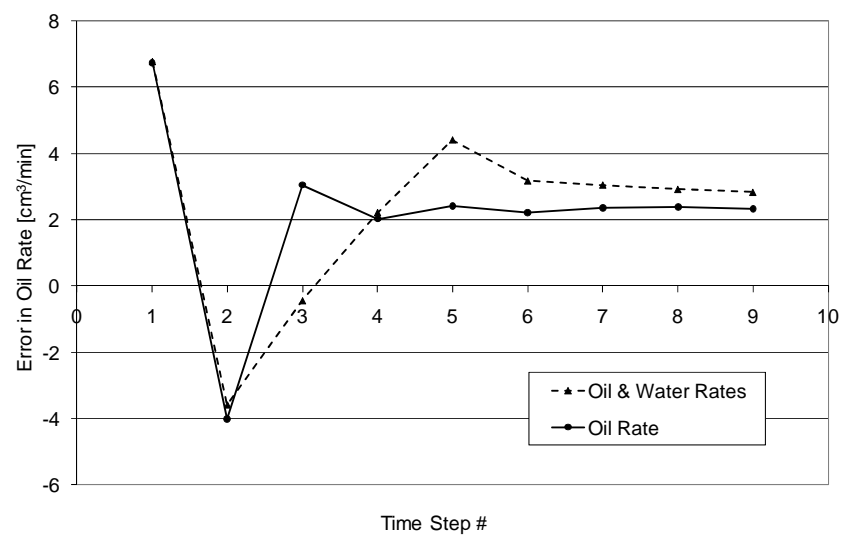

(a)

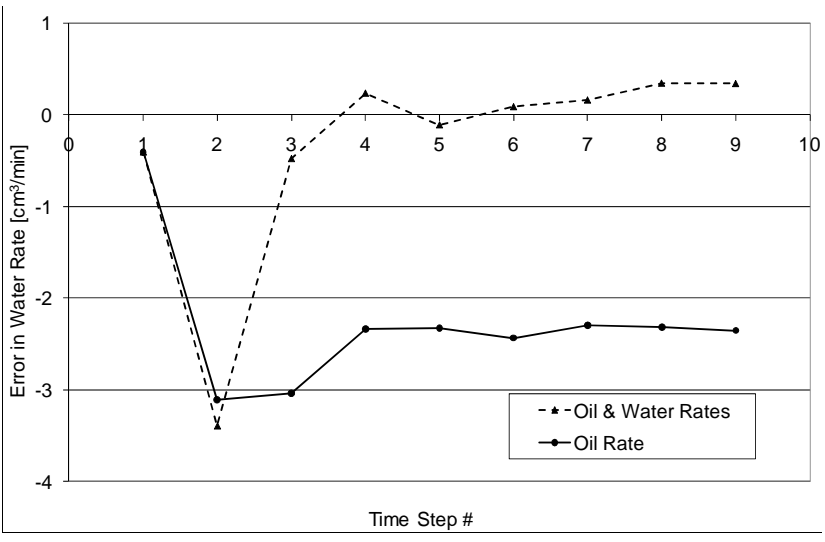

(b)

Figure 11. The total (a) oil and (b) water rates were compared with the actual oil rate at each time step. Updated models with only oil rates (Case 2) resulted in better oil rate prediction than Case 2 . Better water rate prediction was obtained in Case 1 than Case 2.

For total water rate:

$$
E_{w}=\left(\hat{q}_{w}^{P 1}+\hat{q}_{w}^{P 2}\right)-\frac{1}{N R} \sum_{i=1}^{N R} \hat{q}_{w, i}^{P 1}+\hat{q}_{w, i}^{P 2}
$$

The error in the net rate is:

$$
E_{n e t}=E_{o}-E_{w}
$$

Where $\hat{q}_{j}$ is the measured rate of phase $j$. Similar to the synthetic case, Case 2 showed less error in the prediction of oil rates than Case 1. This is because in Case 1, the objective is to reduce the error in both water and oil rates at the expense of increased mismatch in oil rates (Figure 11). Another comparison was looking at the error in the net production (5). The net production can be weighted according to the price of oil and water treatment and included as an objective function for optimization. Figure 12 shows similar errors in the prediction at initial times, but at later times the first case seems to reduce the error systematically while the mismatch for the second case continues deviated. This is because the second case does not explicitly consider the water produc-

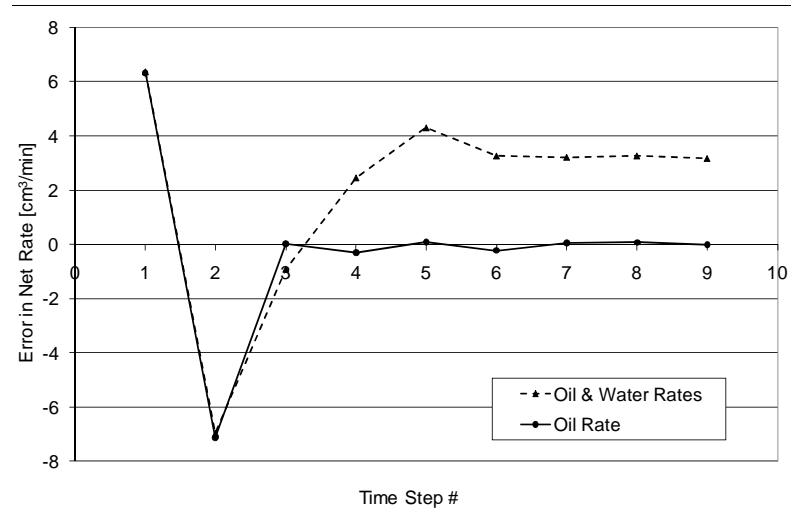

Figure 12. The overall error in the prediction of the net production is similar for both cases at early times. However, at later times the first case gives better predictions in the net production than the second one. 
tion rate as an objective function component.

The permeability maps obtained after updating Cases 1 and 2 are compared in Figure 13. As in the synthetic case, the water rates carried information about the region invaded by water. The permeability distribution in the water leg is therefore updated in the first case where the water rate was included as an objective function component. In Case 2, no major changes occurred in that zone.

The streaks of permeability observed in the map for the first case can be interpreted as local heterogeneities created due to compaction/settling of the coarse glass beads. The resulting permeability map from Case 2 indicates that either the zone invaded by water was very homogeneous (contrary to the Case 1 result) or the oil rate does not contain enough information for updating the model in that region. The synthetic example indicated that the latter explanation is more probable. However, the prediction of streaks of high permeability close to the well needs further investigation and visualization schemes.

\section{Optimal Control from Updated Model}

Once all the permeability realizations are updated using (2), the most probable permeability field or the mean of all realizations is computed. The optimum control settings are developed for this most probable realization. This can be posed as an optimization problem as follows: Find the set of controls (u) that maximizes the cumulative oil production minus the cumulative water production from the current time to the terminal time subject to the water coning equations as constraints. Denoting the objective function as $J$ :

$$
J=\sum_{t}^{T b t}\left(q_{o}^{t} \times \omega_{o}\right) \Delta t+\sum_{T b t}^{T}\left(q_{o}^{t} \times \omega_{o}-q_{w}^{t} \times \omega_{w}\right) \Delta t
$$

$q_{o}$ is the oil rate, $q_{w}$ is the water rate, $T$ is the final time, and $T_{b t}$ is the breakthrough time. $\omega_{o}$ and $\omega_{w}$ are weights assigned to the oil and water production rates respectively, according the revenue or cost associated to them. The breakthrough time $T_{b t}$ can be obtained by integrating the velocity equation found in [9], which requires knowledge of the updated average permeability $k$ around the well. The water oil ratio after breakthrough can be obtained from correlations from literature such as Bournazel and Jeansen's [10] correlation. For water conning problem in the laboratory 2D-flow model, the specified values of $\omega_{o}$ and $\omega_{w}$ are 0.1 and 1 , respectively. These values are arbitrary. The objective function integrates the time discrete oil and water rates from initial time to a pre-set terminal time. This function can be rapidly evaluated without using the flow simulator by calculating the integral from time zero to the breakthrough time and from that time to the terminal time. The breakthrough time is the numerical integral of the velocity expression given by Farmen et al. [9]:

$$
t b t=\int_{0}^{1} \frac{\mathrm{d} h}{\frac{\mu}{2 \pi k D} \ln \left[\frac{r_{o}}{b(1-h)}\right]-\Delta \rho g b h-2 P_{c}}
$$

Every time step, after the model is updated, the objective function is evaluated and maximized for the remaining time using Newton-Raphson method. The average permeability required for the correlations is calculated using the most probable permeability field and the control flow rate $q_{o}$ is assumed constant for the remaining time. Iterative methods require multiple function evaluations, for that reason is convenient to have an analytical solution that can be evaluated without running the reservoir simulator.

\subsection{Model Based Optimization of Production System}

The value of the data assimilation and model updating is capitalized only when the well operating conditions are adjusted to maximize the production of clean oil. This implementation assess the importance of reservoir model updating for establishing optimum control of well production and to demonstrate the complete feedback loop. In this example the flow rate of a vertical well in a $2 \mathrm{D}$

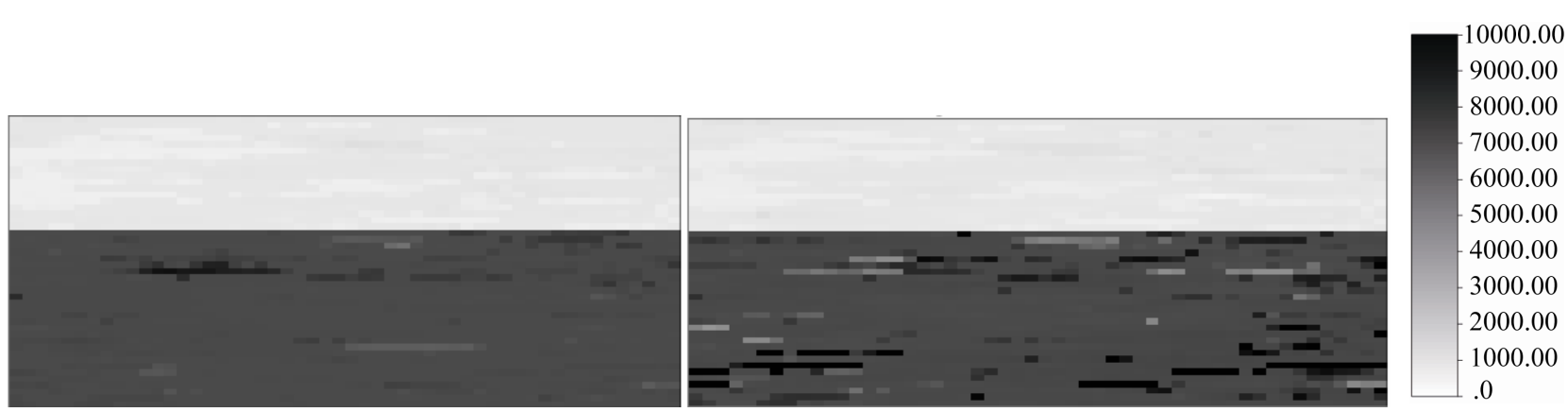

Figure 13. (a) Permeability model obtained constrained only to oil rate (left), and (b) final permeability map of one realization obtained constrained to the measured oil and water rates (right). 


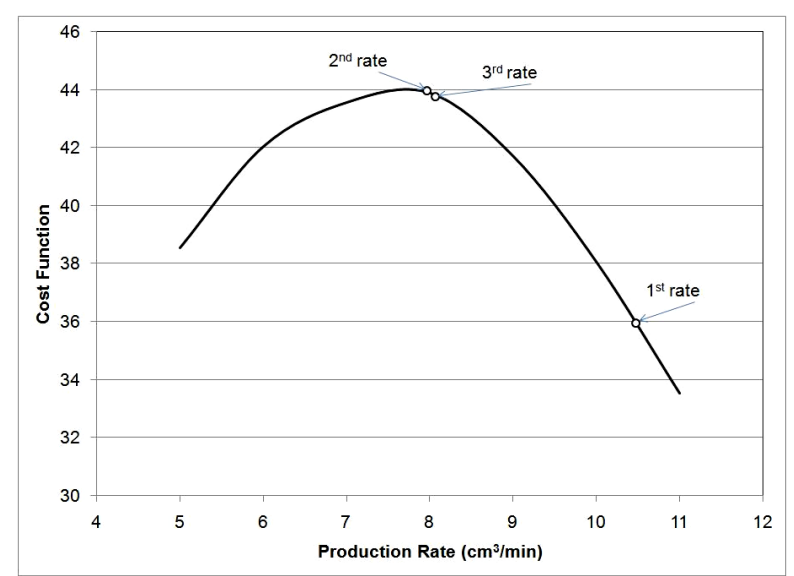

Figure 14. Cost Function plot. The initial flow rate was adjusted such that the cost function was maximized

laboratory size reservoir is optimized as the model is updated. The porous medium is divided into $50 \times 100$ gridblocks $(0.5 \mathrm{~cm} \times 1 \mathrm{~cm})$. The reference permeability values were drawn from a log-normal pdf with $340 \mathrm{mD}$ as mean, and then distributed in the gridblocks using sequential Gaussian simulation forming thin layers. Two water injector wells maintain a constant pressure boundary at the left-hand side of the box; an oil injector is perforated in the top 4 gridblocks part of the model, and a water injector is perforated in the bottom zone. These injectors are placed to mimic constant pressure conditions at the far boundary. On the right edge of the model, a producer well is perforated in the top oil saturated interval and produces at a constant liquid rate. This flow rate constraint is the controllable variable used to optimize the cost function. The initial saturation and pressure distribution is assumed to be known. The reference production data are the bottom-hole pressure, and the water cut of the producer over a pre-determined time interval. The well is assumed to be under constant oil rate control.

After breakthrough, water cut increases until it reaches a plateau at steady state. The correlation for water cut prediction after breakthrough proposed by Bournazel and Jeanson [10] was employed here to evaluate it as function of time. Both breakthrough time and water cut correlations facilitated the fast calculation of the cost function without using the reservoir simulator. A gradient based optimization routine can be easily implemented to find the optimum liquid rate that maximizes the cost function $J$. The initial liquid rate for the reference case is set as the optimum one, based on the initial guess of the permeability distribution.

The cost function plot shown in Figure 14 was constructed by directly running the flow simulator to the final time using different constant flow rates. The initial flow rate $\left(10.47 \mathrm{~cm}^{3} / \mathrm{min}\right)$ was calculated to maximize

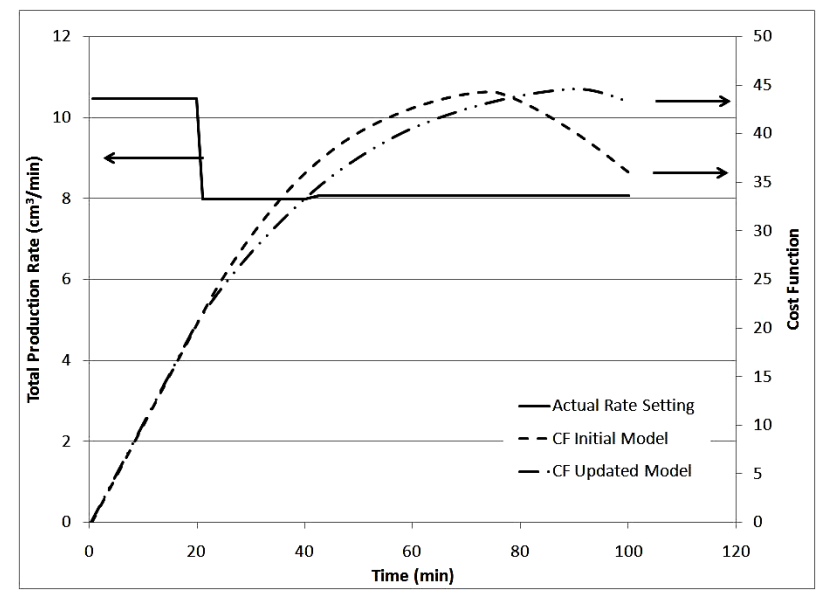

Figure 15. The total liquid rate control was dynamically updated after each time step was assimilated. The final cost function was maximized.

the objective function using the breakthrough time and water-oil ratio correlations according to the initial average permeability, as indicated in Figures $\mathbf{1 4}$ and $\mathbf{1 5}$. However, since the permeability of the model was updated after each time step, a new optimal flow rate was recalculated. After time step 2, the flow rate was recalculated and corrected towards the true optimal. After water breakthrough at time step 3, the rate was moved closer to the true optimal rate. The rate correction at the last time step was minimal because the average permeability of the updated model did not change significantly. This dynamic rate control resulted in a maximization of the objective function as shown in Figure 15. The deviation from the optimum flow rate is due to two reasons: first, the approximations made for calculating the breakthrough time and water cut evolution using correlations and second because of the residual uncertainty in the average permeability. Nevertheless, the flow rate was effectively guided towards the real optimum rate

\section{Conclusions and Further Research}

Oil wells affected by water coning can be managed effectively with the aid of rapid model updating scheme such as Ensemble Kalman Filter in combination with optimal control. The sensitivity analysis on the type and amount of data used to update models shows that models are better updated when both oil and water rates are assimilated using Ensemble Kalman Filter, especially in the aquifer zone. This approach was implemented in a laboratory experiment, confirming the important role of the water rates to update the model in the water invaded zone. Finally, the value added by this feedback control scheme was capitalized when the operating conditions of a vertical well affected by water coning were re-evalu- 
ated after updating prior geological models, using analytical correlations of breakthrough time and water-oil ratio for a fast evaluation and optimization of the objective function.

\section{References}

[1] M. Muskat and R. D. Wyckoff, "An Approximate Theory of Water Coning in Oil Production," Transaction of American Institute of Mining, Metallurgical, and Petroleum Engineers, Vol. 114, 1935, pp. 144-161.

[2] A. Alikan and F. Ali, "State-of-the-Art of Water Coning Modeling and Operation," SPE 13744, Middle East Oil Technical Conference and Exhibition, Barhaim, 1985. doi:10.2118/13744-MS

[3] G. Nævdal, L. Johnsen and V. E. Aanonsen, "Reservoir Monitoring and Continuous Model Updating Using Ensemble Kalman Filter," SPE 75235 Presented at SPE Improved Oil Recovery Symposium, 2003.

[4] Y. Gu and D. Oilver, "The Ensemble Kalman Filter for Continuous Updating of Reservoir Simulation Models," Journal of Energy Resources Technology, Vol 128, No. 1, 2006, pp. 79-87. doi:10.1115/1.2134735

[5] M. Zafari and A. C. Reynolds, "Assessing the Uncer- tainty in Reservoir Description and Performance Predictions with the Ensemble Kalman Filter," Presented at the 2005 SPE Annual Technical Conference and Exhibition, Dallas, 2005. doi:10.2118/95750-MS

[6] G. Evensen, J. Hove, H. C. Meisingset, E. Reiso, K. S. Seim and Espelid, "Using the EnKF for Assisted History Matching of a North Sea Reservoir Model," SPE 106184 Presented at the SPE Reservoir Simulation Symposium, Houston, 2007.

[7] J.-A.Skjervheim, G. Evensen, S. I. Aanonsen, B. O. Ruud and T. A. Johansen, "Incorporating 4D Seismic Data in Reservoir Simulation Models Using Ensemble Kalman Filter," SPE 95789, Presented at the SPE Annual Technical Conference and Exhibition, Dallas, 2005.

[8] G. Evensen, "Sequential Data Assimilation for Non-Linear Dynamics: The Ensemble Kalman Filter," SpringerVerlag, Berlin Heidelberg, 2002.

[9] J. Farmen, G. Wagner, U. Oxaal, P. Meakin and T. Jøssang, "Dynamics of Water Coning," Physical Review E, Vol. 60, No. 4, 1999, pp. 4244-4251. doi:10.1103/PhysRevE.60.4244

[10] C. Bournazel and B. Jeanson, "Fast Water-Coning Evaluation Method," SPE 3628, Proceedings of Fall Meeting of the Society of Petroleum Engineers of AIME, 1971. 